\title{
About the asymmetry structure of the leaf blade Common plantain
}

\author{
Sergey G. Baranov ${ }^{1,}$, Igor E. Zykov², Tatyana S. Biryukova², Lubov V. Fedorova ${ }^{3}$, and Alla \\ M. Antsyshkina ${ }^{3}$ \\ ${ }^{1}$ Vladimir State University named after A.G. and N.G. Stoletov, Gor'kogo, 79, 600005 Russian \\ Federation \\ ${ }^{2}$ State Humanitarian Technological University, 22, Zelenaya Street, 142611 Orekhovo-Zuevo, \\ Russian Federation \\ ${ }^{3}$ I.M. Sechenov First Moscow State Medical University, 8-2 Trubetskaya str., 119991 Moscow, \\ Russian Federation
}

\begin{abstract}
Using the method of geometric morphometrics the contour of the image of the leaf blade of the Common plantain (Plantago major) was aligned along the axis of symmetry to answer the question of whether the asymmetry and shape were influenced by environmental pollution by vehicles and climatic conditions. Procrustes distances showed that fluctuating asymmetry was higher in roadside populations in 2019. In the control populations, a mixture of two types of asymmetry, fluctuating and directional, was obtained. In 2020 with high precipitation (47\% more than in 2019), asymmetry was represented by higher directional asymmetry ( $p$ $<0.0001$ ), although the overall asymmetry remained the same. The nonparametric Kruskal-Wallis test showed the influence on the shape only climatic environments of the year $(p<0.001)$. The geographical location of the populations and the combined effect of the factors year and the place of leaves gathering did not affect the shape of the leaf blade. In 2020, no data were obtained on the excess of asymmetry in roadside populations compared to the control, therefore authors conclude about the weak bioindicative properties of the Common plantain in response to traffic pollution.
\end{abstract}

\section{Introduction}

The high morphological plasticity of plants is a serious cause interferes bioindication on fluctuating asymmetry index. Developmental stability, which is asessed on the basis of undirected, fluctuational deviation from bilateral symmetry, is considered as a genetic and phenotypic property $[1,2,3]$. Some researchers believe that fluctuating asymmetry (FA) is associated with plastic variability and adaptation, that is, FA is included in the adaptation arsenal, but does not serve as a measure of stress. Fluctuating asymmetry refers to a special type of phenotypic variation that depends on genetic properties. To determine the effect: dose - stress - FA, it is important to take into account the stress load gradient and the choice

* Corresponding author: bar.serg58@gmail.com 
of suitable control, as well as careful measurement and checking various types of measurement errors [4]. The studies in which the value of FA is associated with stress exposure from the environment are carried out with various plant species, but they do not always show unequivocal positive results. A large number of publications on the indicative properties of silver birch arouse unremitting interest in the field of fluctuating asymmetry. For example, the influence of the elevation of the relief and the geographical position of populations with climatic features on FA is reported [5-10]. The obstacles are the different genetic status of the studied populations, i.e. different trajectories canalized ontogenetic path and unexplored effects hormesis and paradoxical effect with inadequate reaction to toxins [11]. The development of the methods of geometric morphometrics makes it possible to determine the difference in the coordinates of the left and right halves of the leaf blade. For this, the configuration of the leaf blade samples is aligned along the symmetry axis, and the FA value is determined in two way analysis of variance (Procrustes ANOVA). The proposed work took into account the advantage of this analysis for study the fluctuating asymmetry and shape of the leaf blades Common plantain (Plantago major), a common ruderal plant that can serve as an indicator of environmental pollution. Studies with the measurement of dimensional characteristics have shown that the plantain leaves often possess directional asymmetry [12-13], and the high plasticity of the leaf shape depended on the salinity of the soil and the geographic characteristics of the population area [14]. Exceeding a certain threshold of the FA value indicates a deviation in developmental stability. The relationship between plastic variability and developmental instability has not been sufficiently studied and probably based on the principle of feedback [15-16]. Therefore, a comparison of the shape and asymmetry, using the coordinates of the labels applied to the image of the leaf blades, can help in answering the question the relationship between two types of variability. In the proposed paper, we studied the shape and asymmetry of the leaf plate depending on the pollution of the air along the highways for 2 years with different volume of rainfall in three sites separated by distance $100 \mathrm{~km}$ along the same geographical latitude.

\section{Materials and Methods}

\subsection{Sites and sampling}

Three geographical points were selected, located on the same latitude with a difference of $\mathrm{c}$. one degree and on a distance about $100 \mathrm{~km}$ from each other. One in Vladimirskaya $\left(56^{\circ} 15^{\prime} 81.74^{\prime \prime} \mathrm{N} ; 40^{\circ} 46^{\prime} 58.51^{\prime \prime} \mathrm{E}\right)$ and two in the Moscow region: Orekhovo-Zuevo $\left(55^{\circ} 47^{\prime} 48.4^{\prime \prime} \mathrm{N} ; 39^{\circ} 00^{\prime} 46.8^{\prime \prime} \mathrm{E}\right)$ and the Izmailovo region in Moscow $\left(55^{\circ} 46^{\prime} 46.9^{\prime \prime} \mathrm{N}\right.$; $\left.37^{\circ} 44^{\prime} 01.7^{\prime \prime} \mathrm{E}\right)$.

At each sampling site, the experimental one covered square $50-100 \mathrm{~m} \times 1 \mathrm{~km}$ along the road. The control zone with the same square lied not less than $400 \mathrm{~m}$ from the experimental zone. The sampling areas had fairly uniform physicochemical properties, which could indicate a close trajectory of ontogenetic development. Both zones did not differ in illumination and represented an open area occupied by common ruderal urban vegetation (quinoa, chicory, bluegrass, wheatgrass); the projective cover of plantain was $30-50 \%$. The leaf blades with a half leaf width of $6.0 \pm 0.1 \mathrm{~cm}$, two to three from each of 50 plant, were harvested in August-September 2019-2020 and photographed twice. From each site, 100150 leaf blades were sampled, i.e. a total sum was about 2,000 leaf blades. The calculation of vehicle emissions was carried out according to the standart methodology by the number of vehicles passing along the highways per unit of time. The total emission, taking into 
account all pollutants on the highways, was $0.002 \mathrm{~g} / \mathrm{sec}$ (Orechovo and Moscow) and 0.003 $\mathrm{g} / \mathrm{sec}$ (Vladimir, standart error \pm 0.0001 ).

\section{Measuring and statistics}

We considered both the plant and the leaf blade as a conventional experimental unit. The first two labels applied were not paired and formed an axis of symmetry; the other 25 pair represented the homologous paired traits. Labeling was carried out twice on each image in the JPEG format. The software used (TPS and MorphoJ), as well as recommendations for their use, are freely available on the website of the morphogeometric group https://sbmorphometrics.org. The essence of Procrustes generalized analysis of variance (GPA) is in the constructing a consensus shape, removing the difference in the size of leaf blade samples and in evaluating the difference in the variance of paired labels XY coordinates [17-19]. First, the coordinates of the consensus centroid were determined. Then the difference in shape (classifier 'individual'), the value of FA ('individual $\times$ side'), and directional asymmetry, DA ('side') was statistically determined on the mean F- Goodall ratio, correspondingly $\mathrm{F}_{\mathrm{I} \times \mathrm{S}}$ and $\mathrm{F}_{\mathrm{S}}$.

On the basis of vector coordinates, the covariance matrices of two types were created symmetric matrices and asymmetry matrices. The canonical variate analysis (CVA) was carried out on the data XY coordinates. The symmetry matrix showed the difference in coordinates in the samples among the same landmarks and was used to test the shape of the leaf blade. The asymmetry matrix showed the difference in homologous landmarks between left and right sides and revealed differences in bilateral asymmetry. The difference assumed the distances between the centers of the sets of coordinates, the so-called Procrustes distances. An alternative method was the Kruskal-Wallis analysis. The statistical evaluation used the significance level $\alpha=95 \%$. Permuting multiplication of samples was carried out in 10,000 rounds. The measurement error was calculated in the percentage of the MS value of fluctuating asymmetry.

\section{Results and Discussion}

\subsection{Bilaterall asymmetry}

Procrustes analysis of the total amount of leaves for 2 years showed a high content of fluctuating asymmetry in the whole set of leaves, in control, and in leaves sampled near the road (Table 1).

Table 1. Result of GPA for: a) total, b) control and: c) experiment.

\begin{tabular}{|l|c|c|c|c|}
\hline \multicolumn{1}{|c|}{ Effect } & $S S$ & $M S$ & $d f$ & $F$ \\
\hline a) Individual (all leaves) & 2.55 & 0.02 & 150 & $21.3^{* * *}$ \\
\hline Side & 0.03 & 0.00 & 50 & $0.68^{\text {ns }}$ \\
\hline Ind x side & 0.12 & 0.00 & 150 & $23.53^{* * *}$ \\
\hline Residual & 19.04 & 0.00 & 561200 & \\
\hline b) Individual (control) & 1.24 & 0.02 & 50 & $13.58^{* * *}$ \\
\hline Side & 0.01 & 0.00 & 50 & $0.1^{\text {ns }}$ \\
\hline Ind $\times$ side & 0.09 & 0.00 & 50 & $52.05^{* * *}$ \\
\hline Residual & 10.24 & 0.00 & 293000 & \\
\hline c) Individual (road) & 1.24 & 0.02 & 50 & $68.29^{* * *}$ \\
\hline Side & 0.03 & 0.00 & 50 & $1.57^{\text {ns }}$ \\
\hline Ind $\times$ side & 0.02 & 0.00 & 50 & $11.09^{* * *}$ \\
\hline Residual & 8.81 & 0.00 & 268200 & \\
\hline
\end{tabular}

Notes: ${ }^{* * *}-p<0.0001 ; \mathrm{ns}-p>0.05$ - of square; $d f$ - degree of fredom; $M S$ - mean squre; $F$ - criterion of Goodall 
In all three cases, the consensus size did not differ $(p>0.05)$. Classifier "individual" showed a difference in shape $(p<0.0001)$. The control and experimental leaves showed the same size of consensus $p$-value and significant fluctuating asymmetry without directional asymmetry (the "side" factor is not statistically significant). The value of F-Goodall ratio was higher in control, than in experiment (correspondingly: 52,05 и 11,09). The analysis of populations carried out separately in 2019 and in 2020 showed the next results: in 2019, FA value slightly prevailed in the roadside population, in all cases there was the presence of directional asymmetry (Table 2).

Table 2. Two kids of asymmetry in 2019-2020.

\begin{tabular}{|c|c|c|c|c|c|c|c|c|c|}
\hline \multicolumn{5}{|c|}{ Road } & \multicolumn{5}{|c|}{ Control } \\
\hline \multicolumn{10}{|c|}{2019} \\
\hline Effect & $S S$ & $M S$ & $d f$ & $F$ & Effect & $S S$ & $M S$ & $d f$ & $F$ \\
\hline Side & 0.0001 & 0.0001 & 50 & $1.99 * *$ & Side & 0.04 & 0.001 & 50 & $8.93 * * *$ \\
\hline Ind $\times$ side & 0.0060 & 0.0001 & 100 & $2.0 * * *$ & Ind $\times$ side & 0.01 & 0.001 & 100 & $2.49 * * *$ \\
\hline \multirow[t]{2}{*}{ Residual } & 4.3676 & 0.0001 & 144500 & & Residual & 5.96 & 0.001 & 170100 & \\
\hline & \multicolumn{8}{|c|}{2020} & \\
\hline Side & 0.041 & 0.001 & 50 & $7.49 * *$ & Side & 0.116 & 0.001 & 100 & $16.09 * *$ \\
\hline Ind $\times$ side & 0.011 & 0.000 & 100 & $3.28 * *$ & Ind $\times$ side & 0.062 & 0.001 & 50 & $17.31 * *$ \\
\hline Residual & 4.116 & 0.000 & 123300 & & Residual & 3.920 & 0.000 & 122500 & \\
\hline
\end{tabular}

Notes: ${ }^{* * *}-p<0.0001 ;{ }^{* *}-p<0.001$

In 2020, compared to 2019, the content of directional asymmetry was 3.8 times higher near the road, and 1.8 times higher in the control (see values $\mathrm{F}_{\mathrm{S}}=7.49$ and $\mathrm{F}_{\mathrm{S}}=17.31 ; p$ $<0.0001$ ). The error measure (residuals) was from $0.1 \%$ (control) to $9.01 \%$ (road). Accordingly, the FA value in plants near the road was lower.

Procrustes distances in 2020 between the set of coordinates of control leaves and leaves near the road did not differ (Procrustes distance 0.003; $p>0.05$ ). In 2019, the difference was significant (Procrustes distance $0.005 ; p<0.0001$ ). Thus, the rainy growing season in 2020 reduced the differences in asymmetry. The asymmetry differed in the controlexperiment pair in the summer of 2019, with average precipitation value. The comparison of the two years of follow-up showed that there were no differences in overall asymmetry. This was demonstrated by the diagram of the canonical analysis of variance for the first component CV1 (Fig. 1A). The difference in asymmetry between years was higher (0.008; $p<0.0001)$ than the difference between the total control and all leaves by the road $(0.003 ; p$ $=0,002$ Fig. $1 \mathrm{~B})$. 


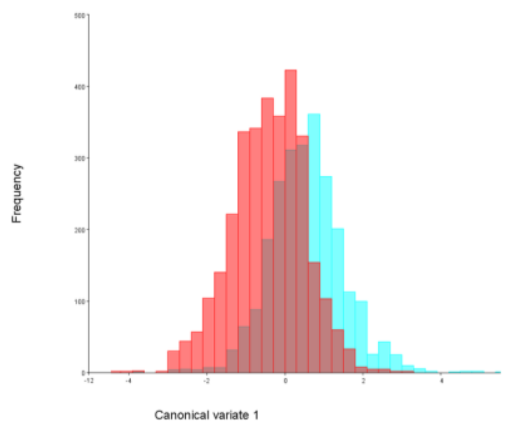

A

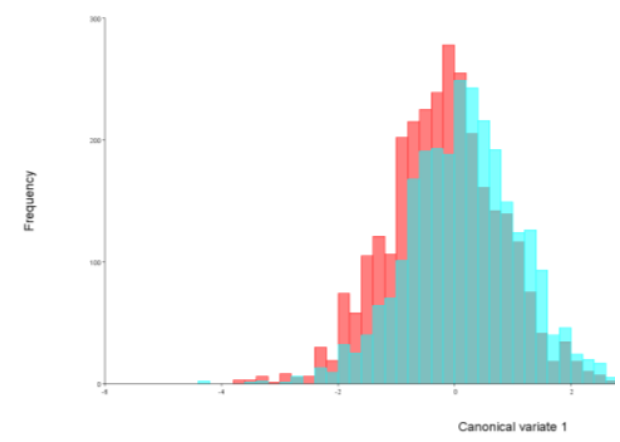

B

Fig. 1. Difference in asymmetry component of shape variation (CV1). A: red color - 2020; cyan color -2019 ; Procrustes distances among groups: $0.008(p<0.0001)$. B: control (red) and road (cyan); Procrustes distances among groups: $0.003(p=0.002)$.

GPA for total set of leaves in 2019 revealed the FA free of DA $\left(\mathrm{F}_{\mathrm{I} \times \mathrm{S}}=10.4 ; p<0.0001\right.$; $\mathrm{F}_{\mathrm{S}}$ was not significant; $\left.p>0.05\right)$. In 2020 there were both type of asymmetry: $\left(\mathrm{F}_{\mathrm{I} \times \mathrm{S}}=3.27 ; p\right.$ $\left.<0.0001 ; \mathrm{F}_{\mathrm{S}}=18.04 ; p<0.0001\right)$. Thus, in a wet year, an increased directional asymmetry was observed, in contrast to a temperate climatic year, where fluctuating asymmetry occupied big dole of the asymmetry. The larger samples neutralized the presence of directional asymmetry. This was true for both years of study. The sample size with $n=50$, even with a fourfold increase in replicas, was clearly insufficient to obtain a representative result. So the plantain leaf blades had a high fluctuating asymmetry, tested only at the maximum sample sizes, which should be taken into account when evaluating the FA and developmental stability.

\subsection{Shape of leaf blade}

Non-parametric Kruskal-Wallis analysis showed the impact of the only factor 'year' on the shape of the leaf blades $(p<0.001)$, a sampling site had no effect on the shape. The differences in Procrustes distances between the centers of the control and experimental sets in 2020 were less high than within the same samples, which indicated accented variability in the morphology of leaf blades in a rainy summer. The symmetric component of shape variation varied pronouncedly from year to year in CVA (Fig. 2).

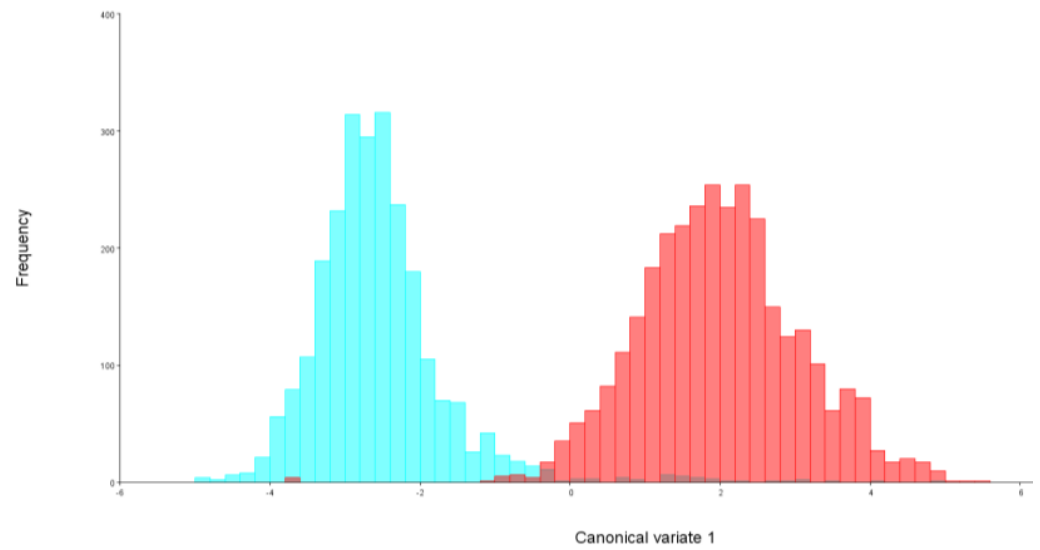

Fig. 2. Symmetric component of shape variation CV1: left -2020 , right -2019 . Procrustes distances among groups: $0.042 ; p<0.0001$. 
In the summer of 2020 the shape of the blade looked oval, and in 2019 the shape was close to the ovoid form with a narrowed proximal. Thus, if in 2019 plantain acted as a reliable bioindicator (75\% of positive results), then in 2020 the results did not allow us to classify this plant as an indicator of developmental stability. This is confirmed by the results obtained on metrical bilateraly symmetrucal traits [13]. Note that bioindication as a property is not so often encountered in recent works, especially concerning the indication of developmental stability by the FA index. The transit of asymmetry from FA to DA, or vice versa, deserves attention as the interaction of genom and phenotype. The opinion about directional asymmetry as a bioindication property for assessing developmental stability remains controversial, although it is known that DA is emerged under stress environment, implying a genotype effect on plant morphology [20]. The high morphological plasticity of this species is the serious reason it could not be considered a reliable indicator with 90$100 \%$ positive results.

A weak correlation was obtained between the two components of the shape $(r=0.13$ $0.14 ; p<0.001)$, i.e. shape has been associated with asymmetric variability. Directional asymmetry, as latent, expressed within high degree of freedom df only at small sample sizes and could depend on the frequency distribution of the sample. For example, the control leaf pool showed directional asymmetry at all level of classifier (individual, leaf and image). Testing of experimental leaves showed statistically significant $\mathrm{FA}\left(\mathrm{F}_{\mathrm{I} \times \mathrm{S}}=5.05 ; p\right.$ $<0.0001)$ with statistically insignificant directional asymmetry. Subsequent testing at the image level revealed directional asymmetry $\left(\mathrm{F}_{\mathrm{S}}=2.4 ; p<0.0001\right)$. Such a "hidden" DA was revealed when testing the asymmetry of woody plants [21-22]. The further research will be reffered to the study of the heterogeneity of the samples distribution. The multivariate factor analysis of shape and asymmetry over several years of research as data accumulates is also important.

\section{Conclusions}

a) Plantain leaf blades had a pronounced fluctuating asymmetry, which was determined with a significant volume of leaves (more then 150 leaf blades).

b) Large plantain, like other herbaceous perennial plants (for example, strawberry species), has dubious bioindication properties on the fluctuating asymmetry index, because climatic factors, for example, the amount of precipitation, have a stronger effect than man-made pollutants.

c) During 2 years of observation, the structure of asymmetric variability, available at high sample sizes, changed from FA to directional asymmetry under high humidity environment. The shape of the leaf plate of Common plantain varied significantly correlating to the total asymmetry which contained two kinds varying components FA and DA.

\section{References}

1. S. Baranov, I. Vinokurov, L. Fedorova, Gene Expression and Phenotypic Traits 2019 Dec 13 (2019)

2. S.V. Dongen, J. Evol. Biol., 19(6) (2006)

3. J.H. Graham, J.M. Emlen, D.C. Freeman, Nonlinear dynamics and developmental instability (2003)

4. N.S. Fox, J.J. Veneracion, J.L. Blois, Ecol. Evol., 10.7 (2020) 
5. E.V. Koroteeva, D.V. Veselkin, N.B. Kuyantseva, O.E. Chashchina, In Doklady Biological Sciences, 460(1) (2015)

6. V. Zverev, A.D. Lama, M.V. Kozlov, Ecological indicators, 1(84) (2018)

7. E. Shadrina, N. Turmukhametova, V. Soldatova, I. Korotchenko, and G. Pervyshina, Symmetry, 12(8) (2020)

8. V.M. Zakharov, E.G. Shadrina, N.V. Turmukhametova, E.N. Ivantsova, E.A. Shikalova, V.Y. Soldatova, I.E. Trofimov, Biol. Bulletin, 47 (2020)

9. D. Miljković, M. Stefanović, S. Orlović, M.S. Neđić, L. Kesić, S. Stojnić. Alpine Botany, 129(2) (2019)

10. J. Brunet and M. L. Van Etten, International Journal of Plant Sciences, 180:9 (2019)

11. E.A. Erofeeva, B.N. Yakimov, Symmetry, 12(5) (2020)

12. M. Veličković D. Tošković. Periodicum biologorum, 117(3) (2015)

13. M.Velickovic, T. Savic, Plant species biology, 27(1) (2012)

14. M.J. Smekens, P.H. van Tienderen, Acta Oecologica, 22(4) (2001)

15. R. M. Doret, J.A. Draghi, and M.C. Whitlock, Evol. Lett., 4(6) (2020)

16. C. P. Klingenberg, Frontiers in Ecology and Evolution 7: 56 (2019)

17. N.S. Fox, J.J. Veneracion, J.L. Blois, Ecology and evolution, 10(7) (2020)

18. V.Viscosi, P. Fortini, D. E. Slice, A. Loy, C. Blasi, Plant Biosystems, 143(3) (2009)

19. S.G. Baranov, I.E. Zykov, T.S. Biryukova, L.V. Fedorova. IOP Conference Series: Earth and Environmental Science, 666(4) (2021)

20. M.V.Veličković, and D. Jakovčev-Todorović, Genetika, 38(2) (2006)

21. S.G. Baranov, Emerging Science Journal, 2(4) (2018)

22. S.G. Baranov, Computer Science On-line Conference (2018) 